\title{
On exponential convergence to a stationary measure for a class of random dynamical systems
}

\author{
Sergei B. Kuksin
}

\begin{abstract}
For a class of random dynamical systems which describe dissipative nonlinear PDEs perturbed by a bounded random kick-force, I propose a "direct proof" of the uniqueness of the stationary measure and exponential convergence of solutions to this measure, by showing that the transfer-operator, acting in the space of probability measures given the Kantorovich metric, defines a contraction of this space.
\end{abstract}

\section{Introduction}

In the papers $[3,4,5]$ my collaborators and I considered a special class of random dynamical systems (RDSs) which describes dissipative nonlinear PDEs (e.g., the 2D Navier-Stokes equations), perturbed by a bounded random kick-force. In [3] we proved that these systems have unique stationary measure, by reducing this problem to the problem of uniqueness of a Gibbs measure for a class of 1D Gibbs systems. In $[4,5]$ we developed a coupling approach to study the systems under discussion. This approach gives a shorter proof of the uniqueness and implies that any solution of the system exponentially fast converges in distribution to the stationary measure.

The goal of this work is to present a "direct proof" of the uniqueness and of the exponential convergence by showing that the transfer-operator, corresponding to an RDS as above and acting in the space of probability measures, given the Kantorovich(-Wasserstein) metric, defines a contraction of this space.

The proof presented in this work can be treated as re-interpreting of the arguments from [4, 5]: it is based on the coupling-approach and uses essentially Lemma 3.2 from [4] (which is the heart of the proof of [4]). In addition to the coupling techniques, we now use some ideas, originated in the works of Kantorovich on the mass-transfer problem in 1940's, see [2, 1].

Due to short size of this paper, we practically do not discuss applications of the results obtained, as well as their relation to works of other mathematicians. For

MSC 2000: 37H99, 35Q30.

Keywords : random dynamical systems, Kantorovich metric, Navier-Stokes equations. 
all this information readers are referred to rather detailed introductions to [3, 4] (post-script files of these works, as well as of [5], can be obtained from the author's web-page www.ma.hw.ac.uk/ ${ }^{\sim}$ kuksin).

We keep notations of $[4,5]$ and for convenience repeat them now:

Notations. We denote by $(\Omega, \mathcal{F}, \mathbb{P})$ and $\left(\Omega^{\prime}, \mathcal{F}^{\prime}, \mathbb{P}^{\prime}\right)$ different probability spaces, and abbreviate them to $\Omega$ and $\Omega^{\prime}$, respectively. All metric spaces are given Borel sigma-algebras. $\mathcal{D}(\cdot)$ signifies the distribution of a random variable.

A Hilbert space $H$ with a norm $\|\cdot\|$ is fixed in this work. We use the following notations for objects, related to $\mathrm{H}$ :

$\mathcal{B}=\mathcal{B}(H)$ - sigma-algebra of Borel subsets of $H$;

$C_{b}$ - the space of bounded continuous functions on $H$, given the sup-norm;

$\mathcal{P}$ - the space of probability Borel measures on $H$;

$\mathcal{P}(A)$ - measures from $\mathcal{P}$, supported by a subset $A \subset H$;

$B(R)$ - the closed ball of radius $R$ in $H$, centred at the origin.

Acknowledgements. This paper is based on my talk at the conference in Plestinles-Grèves (June 2001); I am thankful to the organisers for inviting me there. It was written during my visit to IHES in June, 2001 and was typed there; I sincerely thank the institute for hospitality.

\section{A class of random dynamical systems}

Let $H$ be a Hilbert space with a norm $\|\cdot\|$ and an orthonormal basis $\left\{e_{j}\right\}$, and let $S: H \rightarrow H$ be a continuous map such that $S(0)=0$ and $S$ satisfies some conditions, specified below.

Let $\left\{\eta_{k}, k \in \mathbb{Z}\right\}$ be a sequence of i.i.d. random variables $\Omega \rightarrow H$ of the form

$$
\eta_{k}=\eta_{k}^{\omega}=\sum_{j=1}^{\infty} b_{j} \xi_{j k} e_{j},
$$

where $b_{j} \geq 0$ are constants and $\sum b_{j}^{2}<\infty$. It is assumed that $\left\{\xi_{j k}=\xi_{j k}^{\omega}\right\}$ are independent random variables such that $\left|\xi_{j k}\right| \leq 1$ for all $j, k, \omega$, and

$$
\mathcal{D}\left(\xi_{j k}\right)=p_{j}(r) d r \quad \forall j, k .
$$

Here $p_{1}, p_{2}, \ldots$ are functions of bounded variation, supported by the segment $[-1,1]$, and

$$
\int_{-\varepsilon}^{\varepsilon} p_{j}(r) d r>0 \quad \forall j \geq 1, \quad \varepsilon>0 .
$$

We consider the following random dynamical system (RDS) in $H$ :

$$
u(k)=S(u(k-1))+\eta_{k}=: F_{k}^{\omega}(u(k-1)) \quad k \geq 1 .
$$

This RDS defines a family of Markov chains in $H$ with the transition function

$$
P(k, v, \Gamma)=\mathbb{P}\{u(k) \in \Gamma\}, \quad \Gamma \in \mathcal{B}(H),
$$


where $u(\cdot)=u(\cdot ; v)$ is a solution for $(1.3)$ such that $u(0)=v$. Let $\left\{\mathfrak{S}_{k}\right\}$ and $\left\{\mathfrak{S}_{k}^{*}\right\}$ be the corresponding Markov semigroups, acting in the space $C_{b}$ of bounded continuous functions on $H$, and in the space $\mathcal{P}$ of probability Borel measures, respectively:

$$
\begin{gathered}
\mathfrak{S}_{k} f(v)=\mathbb{E} f(u(k ; v)), \quad f \in C_{b}, \\
\mathfrak{S}_{k}^{*} \mu(\Gamma)=\int_{H} \mathbb{P}\{u(k ; v) \in \Gamma\} \mu(d v), \quad \mu \in \mathcal{P},
\end{gathered}
$$

where $u$ is the solution for (1.3) as above.

For any $v \in H$ and $k=0,1, \ldots$ we abbreviate

$$
\mu_{v}(k)=P(k, v, \cdot)=\mathcal{D}(u(k ; v)) .
$$

Now we impose some assumptions on the map $S$. The "right" ones are given in [4], see there conditions A-C. Below we replace them by shorter and stronger conditions $\mathrm{A}^{\prime}$ ) and $\mathrm{B}^{\prime}$ ). The new conditions hold for the RDS which corresponds to the 2D Navier-Stokes equations (see the example below). The proof of the Main Theorem which we present below works under the conditions A-C but becomes somewhat longer, and the notations become more cumbersome.

$\left.\mathbf{A}^{\prime}\right)$ The map $S$ is Lipschitz uniformly on bounded subsets of $H$, and there exists a positive constant $\gamma_{0}<1$ such that

$$
\|S(u)\| \leq \gamma_{0}\|u\| \quad \forall u \in H .
$$

$\left.\mathbf{B}^{\prime}\right)$ For any $R>0$ there is a sequence $\gamma_{N}(R)>0(N \geq 1)$ which converges to zero as $N \rightarrow \infty$, such that

$$
\left\|Q_{N}\left(S\left(u_{1}\right)-S\left(u_{2}\right)\right)\right\| \leq \gamma_{N}(R)\left\|u_{1}-u_{2}\right\| \quad \text { for all } \quad u_{1}, u_{2} \in B(R) .
$$

Here $Q_{N}$ stands for the orthogonal projector $H \rightarrow \overline{\operatorname{span}}\left\{e_{N}, e_{N+1}, \ldots\right\}$.

Example. Let us consider the 2D Navier-Stokes equations, perturbed by a random kick-force $\eta$ :

$$
\begin{gathered}
\dot{u}-\nu \Delta u+(u \cdot \nabla) u+\nabla p=\eta(t, x) \equiv \sum_{k \in \mathbb{Z}} \eta_{k}(x) \delta(t-k), \\
\operatorname{div} u=0, \quad \int u d x \equiv \int \eta d x \equiv 0 ; \quad x \in \mathbb{T}^{2} .
\end{gathered}
$$

Let $H$ be the $L^{2}$-space of divergence-free vector fields on $\mathbb{T}^{2}$ with zero space-average, and let $\left\{e_{j}\right\}$ be the usual trigonometric basis of $H$. Let us assume that the kicks $\eta_{k}$ are random variables in $H$ having the form (1.1) and satisfying (1.2). Normalising solutions $u(t) \in H$ of (1.5) to be continuous from the right, we observe that the equation can be written in the form (1.3), where $u(k)=u(k, \cdot) \in H, k \in \mathbb{Z}$, and the operator $S$ is the time-one shift along trajectories of the free Navier-Stokes system. The condition $\mathrm{A}^{\prime}$ ) obviously holds with $\gamma_{0}=e^{-\lambda}$, where $\lambda$ is the minimal eigenvalue of $-\nu \Delta$ in $H$. It is also well known that $S$ satisfies $\mathrm{B}^{\prime}$ ), see e.g. [3]. 
A measure $\mu \in \mathcal{P}$ is called a stationary measure for the $\operatorname{RDS}(1.3)$ if $\mathfrak{S}_{k}^{*} \mu=\mu$ for all $k$. The goal of this work is to prove the following result:

Theorem 1. There exists a constant $N \geq 1$ such that if

$$
b_{j} \neq 0 \quad \forall j \leq N,
$$

then the RDS (1.3) has a unique stationary measure $\mu$. Moreover, there exists a constant $\kappa \in(0,1)$ such that

$$
\left|\left(\mu_{u}(t), f\right)-(\mu, f)\right| \leq C \kappa^{t} \quad \text { for } \quad t=1,2, \ldots,
$$

for every Lipschitz function $f$ on $H$ such that $|f| \leq 1$ and $\operatorname{Lip} f \leq 1$. The constant $C$ depends only on $\|u\|$.

\section{Preliminaries}

\subsection{Estimates for solutions.}

Since $\left|\xi_{j k}\right| \leq 1$, then

$$
\left\|\eta_{k}^{\omega}\right\| \leq K_{1}=\left(b_{1}^{2}+b_{2}^{2}+\ldots\right)^{1 / 2}<\infty \text { for all } k \text { and } \omega .
$$

So

$$
\left\|F_{k}^{\omega}(u)\right\| \leq \gamma_{0}\|u\|+K_{1}
$$

and any ball $B(R)$ with $R \geq K_{1} /\left(1-\gamma_{0}\right)$ is invariant for the RDS (1.3) (a set $A \subset \mathcal{B}(H)$ is said to be invariant for (1.3) if $P(k, u, A)=1$ for $k \geq 0$ and $u \in A$ ). The same estimate above implies that

$$
\|u(k ; v)\| \leq \gamma_{0}^{k}\|v\|+K_{1}\left(1+\cdots+\gamma_{0}^{k-1}\right) \leq \gamma_{0}^{k}\|v\|+\frac{K_{1}}{1-\gamma_{0}},
$$

for all $k \geq 0, v \in H$ and all $\omega$.

\subsection{The coupling.}

Let $\mu_{1}, \mu_{2} \in \mathcal{P}$.

Definition. A pair of random variables $\xi_{1}, \xi_{2}$, defined on the same probability space and valued in $H$, is called a coupling for $\left(\mu_{1}, \mu_{2}\right)$ if $\mathcal{D} \xi_{1}=\mu_{1}$ and $\mathcal{D} \xi_{2}=\mu_{2}$.

For basic results on the coupling see [6] and Appendix in [4].

The following lemma, proved in [4], Lemma 3.2, claims that measures $\mu_{u_{1}}(1)$, $\mu_{u_{2}}(1)$ admit a coupling which possesses some special properties if $\left\|u_{1}-u_{2}\right\| \ll 1$.

Let us take any $R \geq 1$.

Lemma 1. There is a probability space $(\Omega, \mathcal{F}, \mathbb{P})$, an integer $N=N(R) \geq 1$ and a constant $C_{*}=C_{*}(R)>0$ such that if (1.6) holds, then for any $u_{1}, u_{2} \in B(R)$ the measures $\mu_{u_{1}}(1), \mu_{u_{2}}(1)$ admit a coupling $\left(V_{1}, V_{2}\right), V_{j}=V_{j}\left(u_{1}, u_{2} ; \omega\right)$, with the following properties:

(i) the maps $V_{1}, V_{2}: B(R)^{2} \times \Omega \rightarrow H$ are measurable;

(ii) denoting $d=\left\|u_{1}-u_{2}\right\|$, we have

$$
\mathbb{P}\left\{\left\|V_{1}-V_{2}\right\| \geq d / 2\right\} \leq C_{*} d .
$$




\subsection{A metric on $\mathcal{P}$.}

Let us take any number

$$
R^{\prime}>K_{1} /\left(1-\gamma_{0}\right)
$$

We fix it from now on and abbreviate $B\left(R^{\prime}\right)=B$. Due to the results of section 2.1, the set $B$ is invariant for the $\operatorname{RDS}(1.3)$. Next we take any $\gamma_{1} \in\left(\gamma_{0}, 1\right)$ and any positive $d_{0}$ such that

$$
d_{0} \leq \min \left\{\frac{1}{4 C_{*}}, \frac{1-\gamma_{1}}{2 C_{*}}, 1\right\}
$$

where $C_{*}=C_{*}\left(R^{\prime}\right)$ (see Lemma 1 ). For $k \in \mathbb{Z}$ we set $d_{k}=\gamma_{1}^{k} d_{0}$. We may assume that $d_{0}$ and $R^{\prime}$ are chosen such that $d_{-L}=R^{\prime}$ for some $L \geq 1$. Below we consider the numbers $d_{k}$ with $k \geq-L$ only.

Let us introduce in the space $H$ equivalent metric $d$ :

$$
d\left(u_{1}, u_{2}\right)=\left\|u_{1}-u_{2}\right\| \wedge d_{0},
$$

and consider the set $\mathcal{O} \subset C_{b}$, formed by all functions $f$ such that

$$
\left|f\left(u_{1}\right)-f\left(u_{2}\right)\right| \leq d\left(u_{1}, u_{2}\right) \quad \text { for all } u_{1}, u_{2} .
$$

Clearly,

$$
\frac{1}{2} d_{0} f \in \mathcal{O} \quad \text { if }|f| \leq 1 \text { and } \operatorname{Lip} f \leq 1 .
$$

For any two measures $\mu_{1}, \mu_{2} \in \mathcal{P}$ we define the Kantorovich distance $d_{K}\left(\mu_{1}, \mu_{2}\right)$ as

$$
d_{K}\left(\mu_{1}, \mu_{2}\right)=\sup _{g \in \mathcal{O}}\left\{\left(\mu_{1}-\mu_{2}, g\right)\right\} .
$$

It is known that the space $\mathcal{P}$ is complete with respect to this distance (see [2], [1]), and it is easy to see that $\mathcal{P}(B)$ is a closed subset of $\mathcal{P}$.

We remind that the set $B=B\left(R^{\prime}\right)$ is invariant for (1.3).

Lemma 2. Suppose that there exists a sequence $\zeta_{k} \rightarrow 0$ such that for $k \geq 1$ and $u, v \in B$ we have $d_{K}\left(\mu_{u}(k), \mu_{v}(k)\right) \leq \zeta_{k}$. Then there exists a unique measure $\mu \in$ $\mathcal{P}(B)$, such that

$$
d_{K}\left(\mu_{u}(k), \mu\right) \leq \zeta_{k} \quad \text { for } k \geq 1 \text { and } u \in B
$$

Proof. Let us take any function $f \in \mathcal{O}$. Using the Chapman-Kolmogorov relation and the assumption of the lemma, for $\ell \geq k \geq 0$ and $u, v \in B$ we have:

$$
\begin{array}{r}
\left(\mu_{v}(\ell)-\mu_{u}(k), f\right)=\int_{B} P(\ell-k, v, d z) \int_{B}(P(k, z, d w)-P(k, u, d w)) f(w) \\
\leq \zeta_{k} \int_{B} P(\ell-k, v, d z)=\zeta_{k} .
\end{array}
$$

Hence, $d_{K}\left(\mu_{v}(\ell), \mu_{u}(k)\right) \leq \zeta_{k}$. Since the space $\left(\mathcal{P}, d_{K}\right)$ is complete, then there exists a unique measure $\mu \in \mathcal{P}$ such that $d_{K}\left(\mu_{u}(k), \mu\right) \rightarrow 0$ as $k \rightarrow \infty$, for every $u \in B$. Passing to limit in (2.8) as $\ell \rightarrow \infty$ we recover (2.7). It is clear that $\operatorname{supp} \mu \subset B$. So $\mu \in \mathcal{P}(B)$ and the lemma is proved. 


\section{A Kantorovich-type functional}

First we shall construct a special bounded measurable function $f_{K}$ on $B \times B$, vanishing on the diagonal. To define the function, we consider partition of $B \times B$ to sets $Q_{\ell},-L \leq \ell \leq \infty$. Here $Q_{\infty}$ is the diagonal of $B \times B$,

$$
Q_{r}=\left\{\left(u_{1}, u_{2}\right) \in B \times B \mid d_{r+1}<\left\|u_{1}-u_{2}\right\| \leq d_{r}\right\}
$$

if $0 \leq r<\infty$, and

$$
Q_{r}=\left\{\left(u_{1}, u_{2}\right) \in B \times B \mid\left\|u_{1}-u_{2}\right\|>d_{0}, \quad \frac{1}{2} \gamma_{1} d_{r}<\left\|u_{1}\right\| \vee\left\|u_{2}\right\| \leq \frac{1}{2} d_{r}\right\}
$$

if $-L \leq r<0$.

Now we define the function $f_{K}$ :

$$
f_{K}\left(u_{1}, u_{2}\right)= \begin{cases}d_{r}, & \text { if }\left(u_{1}, u_{2}\right) \in Q_{r}, 0 \leq r \leq \infty \\ \widetilde{d}_{\ell}, & \text { if }\left(u_{1}, u_{2}\right) \in Q_{\ell}, \ell<0\end{cases}
$$

where $d_{\infty}=0$ and the numbers $\left\{\widetilde{d}_{\ell}\right\}$ such that

$$
d_{0} \leq \widetilde{d}_{-1} \leq \ldots \leq \widetilde{d}_{-L}
$$

are constructed below. Clearly,

$$
d_{-L} \geq f_{K}\left(u_{1}, u_{2}\right) \geq d\left(u_{1}, u_{2}\right)
$$

for all $u_{1}, u_{2}$.

For any pair of measures $\mu_{1}, \mu_{2} \in \mathcal{P}(B)$ we define a Kantorovich-type functional $\mathcal{K}\left(\mu_{1}, \mu_{2}\right)$ as follows:

$$
\mathcal{K}\left(\mu_{1}, \mu_{2}\right)=\inf \left\{\mathbb{E} f_{K}\left(U_{1}, U_{2}\right)\right\}
$$

where the infimum is taken over all couplings $\left(U_{1}, U_{2}\right)$ for $\left(\mu_{1}, \mu_{2}\right)$.

Everywhere below (and in Theorem 1) $N=N\left(R^{\prime}\right)$ is the constant from Lemma 1.

Theorem 2. Let us assume that the assumption (1.6) holds. Then there exists $\kappa<1$ such that

$$
\mathcal{K}\left(\mathfrak{S}_{1}^{*}\left(\mu_{1}\right), \mathfrak{S}_{1}^{*}\left(\mu_{2}\right)\right) \leq \kappa \mathcal{K}\left(\mu_{1}, \mu_{2}\right)
$$

for all $\mu_{1}, \mu_{2} \in \mathcal{P}(B)$ (provided that the numbers $\widetilde{d}_{-1}, \ldots, \widetilde{d}_{-L}$ are chosen accordingly).

The theorem is proved in the next section. Now we continue to study the RDS (1.3), taking the theorem for granted.

Let $\left(U_{1}, U_{2}\right)$ be a coupling for $\left(\mu_{1}, \mu_{2}\right)$. Using (3.2), for any $g \in \mathcal{O}$ we get:

$$
\left(\mu_{1}-\mu_{2}, g\right)=\mathbb{E}\left(g\left(U_{1}\right)-g\left(U_{2}\right)\right) \leq \mathbb{E} d\left(U_{1}, U_{2}\right) \leq \mathbb{E} f_{K}\left(U_{1}, U_{2}\right) .
$$

Comparing this estimate with the definitions (2.6) and (3.3) we find that ${ }^{1}$

$$
d_{K}\left(\mu_{1}, \mu_{2}\right) \leq \mathcal{K}\left(\mu_{1}, \mu_{2}\right)
$$

\footnotetext{
${ }^{1} \mathrm{~A}$ celebrated theorem of Kantorovich says that the inequality (3.5) transforms to the equality if in $(3.3)$ we replace $f\left(U_{1}, U_{2}\right)$ by $d\left(U_{1}, U_{2}\right)$. See in $[1,2]$.
} 
Let us take any $u_{1}, u_{2} \in B$. Then $\mu_{u_{1}}(k), \mu_{u_{2}}(k) \in \mathcal{P}(B)$ for all $k \geq 0$. Iterating (3.4) and using (3.5) together with the first inequality in (3.2), we obtain

$$
\begin{aligned}
d_{K}\left(\mu_{u_{1}}(k), \mu_{u_{2}}(k)\right) & \leq \mathcal{K}\left(\mu_{u_{1}}(k), \mu_{u_{2}}(k)\right) \\
& \leq \kappa^{k} \mathcal{K}\left(\mu_{u_{1}}(0), \mu_{u_{2}}(0)\right) \\
& =\kappa^{k} f_{K}\left(u_{1}, u_{2}\right) \leq \kappa^{k} \widetilde{d}_{-L} .
\end{aligned}
$$

Applying Lemma 2 we get that there exists a unique measure $\mu \in \mathcal{P}(B)$ such that $d_{K}\left(\mu_{u}(k), \mu\right) \leq \kappa^{k} \widetilde{d}_{-L}$ for all $k \geq 0, u \in B$.

Let us take a measure $\nu \in \mathcal{P}(B)$. For a function $f \in \mathcal{O}$ we have:

$$
\left(\mathfrak{S}_{k}^{*}(\nu)-\mu, f\right)=\int\left(\mu_{u}(k)-\mu, f\right) d \nu(u) \leq \kappa^{k} \widetilde{d}_{-L}
$$

Hence,

$$
d_{K}\left(\mathfrak{S}_{k}^{*}(\nu), \mu\right) \leq \kappa^{k} \widetilde{d}_{-L} \quad \forall k \geq 0, \quad \nu \in \mathcal{P}(B) .
$$

Now let us take any $u \in H$. Due to (2.2) there exists $\ell=\ell(\|u\|)$ such that $\mu_{u}(\ell) \in \mathcal{P}(B)$. Since $\mu_{u}(k+\ell)=\mathfrak{S}_{k}^{*} \mu_{u}(\ell)$, then denoting $k+\ell=t$ we get from (3.6) that

$$
d_{K}\left(\mu_{u}(t), \mu\right) \leq \kappa^{t-\ell} \widetilde{d}_{-L},
$$

for any $u \in H$, where $\ell=\ell(\|u\|)$. Due to (2.5) and (2.6) with $g= \pm \frac{d_{0}}{2} f$, (3.7) implies (1.7) with $C=\widetilde{d}_{-L} \kappa^{-\ell}$.

The estimate (1.7) easily implies that $\mu$ is the unique stationary measure. Indeed, if $\widetilde{\mu}$ is another one, then for any function $f$ as in (1.7) we have

$$
\begin{aligned}
|(\widetilde{\mu}, f)-(\mu, f)|=\mid \int\left(\mu_{u}(k), f\right) \widetilde{\mu}(d u)-\int(\mu, f) & \widetilde{\mu}(d u) \mid \\
& \leq \int\left|\left(\mu_{u}(k)-\mu, f\right)\right| \widetilde{\mu}(d u) .
\end{aligned}
$$

The integrand is bounded by two and goes to zero as $k \rightarrow \infty$ due to (1.7). So the integral goes to zero as $k \rightarrow \infty$ as well and $(\widetilde{\mu}, f)=(\mu, f)$ for all functions as above. Hence, $\mu=\widetilde{\mu}$.

Theorem 1 is proved.

\section{Proof of Theorem 2}

Let us take any $A^{\prime}>\mathcal{K}\left(\mu_{1}, \mu_{2}\right)$. Then there exists a coupling $\left(U_{1}^{\prime}, U_{2}^{\prime}\right)$ for $\left(\mu_{1}, \mu_{2}\right)$ such that $\mathbb{E} f_{K}\left(U_{1}^{\prime}, U_{2}^{\prime}\right) \leq A^{\prime}$. The random variables $U_{1}^{\prime}, U_{2}^{\prime}$ are defined on some probability space $\left(\Omega^{\prime}, \mathcal{F}^{\prime}, \mathbb{P}^{\prime}\right)$. Since supports of $\mu_{1}, \mu_{2}$ belong to $B$, we may assume that $U_{1}^{\prime}, U_{2}^{\prime} \in B$ for all $\omega^{\prime}$. Now applying Lemma 1 with $R=R^{\prime}$, we find measurable maps $V_{1}, V_{2}: B^{2} \times \Omega \rightarrow H$ such that

$$
\mathcal{D}\left(V_{j}\left(u_{1}, u_{2} ; \cdot\right)\right)=\mu_{u_{j}}(1)=P\left(1, u_{j}, \cdot\right)
$$


for $j=1,2$. Let us consider the following random variables $U_{1}, U_{2}$, defined on the probability space $\Omega \times \Omega^{\prime}$ :

$$
U_{j}\left(\omega, \omega^{\prime}\right)=V_{j}\left(U_{1}^{\prime}\left(\omega^{\prime}\right), U_{2}^{\prime}\left(\omega^{\prime}\right) ; \omega\right), \quad j=1,2 .
$$

Let us take any $f \in C_{b}$. Using (4.1) and the fact that $\mathcal{D}\left(U_{1}^{\prime}\right)=\mu_{1}$, we get:

$$
\begin{aligned}
\mathbb{E}^{\omega, \omega^{\prime}} f\left(U_{1}\right) & =\mathbb{E}^{\omega^{\prime}}\left[\mathbb{E}^{\omega} f\left(V_{1}\left(U_{1}^{\prime}\left(\omega^{\prime}\right), U_{2}^{\prime}\left(\omega^{\prime}\right) ; \omega\right)\right)\right] \\
& =\mathbb{E}^{\omega^{\prime}} \int P\left(1, U_{1}^{\prime}\left(\omega^{\prime}\right), d u\right) f(u) \\
& =\int \mu_{1}(d v) \int P(1, v, d u) f(u) \\
& =\left(\mathfrak{S}_{1}^{*}\left(\mu_{1}\right), f\right) .
\end{aligned}
$$

Therefore, $\mathcal{D}\left(U_{1}\right)=\mathfrak{S}_{1}^{*}\left(\mu_{1}\right)$. Similar $\mathcal{D}\left(U_{2}\right)=\mathfrak{S}_{1}^{*}\left(\mu_{2}\right)$, so $\left(U_{1}, U_{2}\right)$ is a coupling for $\left(\mathfrak{S}_{1}^{*}\left(\mu_{1}\right), \mathfrak{S}_{1}^{*}\left(\mu_{2}\right)\right)$.

If we can prove that

$$
\mathbb{E}^{\omega} f_{K}\left(V_{1}\left(u_{1}, u_{2} ; \omega\right), V_{2}\left(u_{1}, u_{2} ; \omega\right)\right) \leq \kappa f_{K}\left(u_{1}, u_{2}\right)
$$

for all $u_{1}, u_{2} \in B$, then

$$
\begin{aligned}
\mathbb{E} f_{K}\left(U_{1}, U_{2}\right)=\mathbb{E}^{\omega^{\prime}}\left[\mathbb{E}^{\omega} f_{K}\left(V_{1}\left(U_{1}^{\prime}, U_{2}^{\prime} ; \omega\right), V_{2}\left(U_{1}^{\prime}, U_{2}^{\prime} ; \omega\right)\right)\right] & \\
& \leq \kappa \mathbb{E}^{\omega^{\prime}} f_{K}\left(U_{1}^{\prime}, U_{2}^{\prime}\right) \leq \kappa A^{\prime} .
\end{aligned}
$$

So $\mathcal{K}\left(\mathfrak{S}_{1}^{*}\left(\mu_{1}\right), \mathfrak{S}_{1}^{*}\left(\mu_{2}\right)\right) \leq \kappa A^{\prime}$ and (3.4) would follow since $A^{\prime}$ is an arbitrary number bigger than $\mathcal{K}\left(\mu_{1}, \mu_{2}\right)$. It remains to check (4.2).

Let us find $k \in[-L, \infty]$ such that $\left(u_{1}, u_{2}\right) \in Q_{k}$. If $k=\infty$, then $u_{1}=u_{2}$, so $V_{1}=V_{2}$ and (4.2) holds trivially. Now let $0 \leq k<\infty$. Then, due to (2.3),

$$
\mathbb{P}\left\{\left(V_{1}, V_{2}\right) \in \bigcup_{r \geq k+1} Q_{r}\right\} \geq 1-C_{*} d_{k} .
$$

Since $f_{K} \leq d_{k+1}$ for $\left(V_{1}, V_{2}\right) \in \bigcup_{r \geq k+1} Q_{r}$ and $f_{K} \leq \sup f_{K}=\widetilde{d}_{-L}$ for all $\left(V_{1}, V_{2}\right)$, then

$$
\mathbb{E} f_{K}\left(V_{1}, V_{2}\right) \leq d_{k+1}\left(1-C_{*} d_{k}\right)+\widetilde{d}_{-L} C_{*} d_{k} .
$$

As $f_{K}\left(u_{1}, u_{2}\right)=d_{k}$, then in this case

$$
\frac{\mathbb{E} f_{K}\left(V_{1}, V_{2}\right)}{f_{K}\left(u_{1}, u_{2}\right)} \leq \gamma_{1}\left(1-C_{*} d_{k}\right)+C_{*} \widetilde{d}_{-L}
$$

Therefore, (4.2) holds with some $k$-independent $\kappa<1$ if

$$
C_{*} \widetilde{d}_{-L} \leq 1-\gamma_{1}
$$

If $-L \leq k \leq-1$, then $\left\|u_{1}\right\|,\left\|u_{2}\right\| \leq \frac{1}{2} d_{k}$ and $\left\|S\left(u_{j}\right)\right\| \leq \gamma_{0} \frac{1}{2} d_{k}$ for $j=1,2$. As $d_{k}>d_{0}, \gamma_{0}<\gamma_{1}$ and the random variable $\eta$ with a positive probability is smaller than any fixed positive constant (see (1.2)), then

$$
\mathbb{P}\left\{\left\|V_{1}\right\|,\left\|V_{2}\right\| \leq \frac{1}{2} d_{k+1}\right\} \geq \theta>0 .
$$


If $k \leq-2$, then this means that

$$
\mathbb{P}\left\{\left(V_{1}, V_{2}\right) \in \bigcup_{r \geq k+1} Q_{r}\right\} \geq \theta .
$$

Since $f \leq \widetilde{d}_{-L}$, then we have

$$
\mathbb{E} f_{K}\left(V_{1}, V_{2}\right) \leq \theta \widetilde{d}_{k+1}+(1-\theta) \widetilde{d}_{-L}
$$

As $f_{K}\left(u_{1}, u_{2}\right)=\widetilde{d}_{k}$, then (4.2) holds for $-L \leq k \leq-2$ if

$$
\theta \widetilde{d}_{k+1}+(1-\theta) \widetilde{d}_{-L}=\kappa \widetilde{d}_{k}
$$

If $k=-1$, then for any $\omega$ from the event in the l.h.s of (4.5) we have $\left\|V_{1}\right\|,\left\|V_{2}\right\| \leq$ $\frac{1}{2} d_{0}$. Therefore $\left\|V_{1}-V_{2}\right\| \leq d_{0}$ and $\left(V_{1}, V_{2}\right) \in \bigcup_{r \geq 0} Q_{r}$. So the relation (4.6) still holds for $k=-1$ if we denote

$$
\widetilde{d}_{0}=d_{0}
$$

With this choice of $\widetilde{d}_{0},(4.2)$ holds for all negative $k$ if so does (4.7).

The relations (4.7) are equivalent to

$$
\widetilde{d}_{-L+1}=\frac{\kappa+\theta-1}{\theta} \widetilde{d}_{-L}
$$

and

$$
\widetilde{d}_{-L+r}=\frac{1}{\theta}\left(\kappa \widetilde{d}_{-L+r-1}-(1-\theta) \widetilde{d}_{-L}\right)
$$

for $r \geq 2$. That is,

$$
\widetilde{d}_{-L+r}=\frac{\widetilde{d}_{-L}}{\theta}\left[\left(\frac{\kappa}{\theta}\right)^{r-1}\left(\kappa+\theta-1-\frac{\theta(1-\theta)}{\kappa-\theta}\right)+\frac{\theta(1-\theta)}{\kappa-\theta}\right]
$$

for $1 \leq r \leq L-1$.

Let us assume that $\kappa=1-\varepsilon$, where $0<\varepsilon \ll 1$. Then

$$
\widetilde{d}_{-L+r}=\frac{\widetilde{d}_{-L}}{\theta}\left[\left(\frac{-\varepsilon}{\theta^{r-1}(1-\theta)}+O\left(\varepsilon^{2}\right)\right)+\frac{\theta(1-\theta)}{1-\theta-\varepsilon}\right],
$$

where $O\left(\varepsilon^{2}\right)$ depends on $r \leq L$. Choosing $\varepsilon=\varepsilon_{L}$ sufficiently small, we see that the numbers $\widetilde{d}_{-L+r}(0 \leq r \leq L)$ decay when $r$ grow; so they satisfy all relations in (3.1) (if $\widetilde{d}_{0}=d_{0}$ ).

We have seen that a function $f_{K}$, constructed using the numbers $\left\{\widetilde{d}_{\ell}\right\}$ as above, satisfies (4.2) and (3.1) if it satisfies (4.4) and if $\widetilde{d}_{0}=d_{0}$. Due to (4.8), $\widetilde{d}_{-L}=\widetilde{d}_{0}(1+$ $O(\varepsilon))$. Taking $\widetilde{d}_{0}=d_{0}$, we have $\widetilde{d}_{-L}=d_{0}(1+O(\varepsilon))$. Due to $(2.4), d_{0} \leq\left(1-\gamma_{1}\right) / 2 C_{*}$. So (4.4) is satisfied if $\varepsilon$ is sufficiently small.

We have constructed constants $\widetilde{d}_{k}$ such that the corresponding function $f_{K}$ satisfies (3.4) with some $\kappa=1-\varepsilon<1$. The theorem is proved. 


\section{References}

[1] R. Dudley, Real analysis and probability, Wadsworth\&Brooks/Cole, 1989.

[2] L. Kantorovich, G.Akilov, Functional analysis (in Russian). Moscow, Nauka, 1977.

[3] S. Kuksin, A. Shirikyan, Stochastic dissipative PDEs and Gibbs measure, Commun. Math. Phys. 213 (2000), 291-330.

[4] S. Kuksin, A. Shirikyan, A coupling approach to randomly forced nonlinear PDEs. 1, to appear in Commun. Math. Phys.

[5] S. Kuksin, A. Piatnitskit, A. Shirikyan, A coupling approach to randomly forced nonlinear PDEs. 2, preprint (April, 2001).

[6] T. Lindvall, Lectures on the Coupling Methods, New York, John Willey \& Sons, 1992.

Department of Mathematics

Heriot-WATt University Riccarton, EDINBURGH EH14 4AS U.K.

kuksin@ma.hw.ac.uk

www.ma.hw.ac.uk/ ${ }^{\sim}$ kuksin/ 